\title{
THE PERCEPTION OF AMBIGUOUS IMAGES AS A QUANTUM INFORMATION PROCESS
}

\author{
GIUSEPPE CAGLIOTI (*), GIORGIO BENEDEK (**), \\ LUIGI COCCHIARELLA $(* * *)$
}

Nota presentata dal m.e. Giuseppe Caglioti

(Adunanza dell'8 maggio 2014)

\begin{abstract}
SuNTO. - Viene formulata una simulazione della percezione dinamica del cubo Necker in termini di un sistema quantistico a due livelli - ad esempio un qubit realizzato da una giunzione Josephson o da un elettrone (spin $1 / 2$ ) controllato da campi magnetici -. L'analogia così stabilita indica che la trasformazione neurofisiologica che conduce, mediante una riduzione simmetria, all'inversione percettiva delle due facce del cubo è controllata dai principi della meccanica quantistica. Un valore dell'ordine di 0,32 mJ.s è stimato per l'azione neuro-fisiologica elementare che simula la costante di Planck a livello mesoscopico. In questo studio interdisciplinare sono utilizzati concetti chiave: la simmetria, la riduzione simmetria, l'auto-organizzazione, il principio di sovrapposizione e l'ambiguità.
\end{abstract}

$$
* * *
$$

ABSTRACT. - A simulation of the dynamic perception of the Necker cube in terms of a two level quantum system - e.g. a qubit realized by a Josephson junction or by an electron of spin $1 / 2$ controlled by magnetic fields - indicates that the neuro-physiological

(*) Dipartimento di Energia, Politecnico di Milano, Campus Leonardo, Via Ponzio 34, 20133 Milano, Italy.

E-mail: giuseppe.caglioti@polimi.it

(**) Dipartimento di Scienza dei Materiali, Università di Milano-Bicocca, Via R. Cozzi 55, 20125 Milano, Italy.

E-mail: giorgio.benedek@unimib.it

(min) Dipartimento di Architettura e Studi Urbani, Politecnico di Milano, Via Bonardi 3, 20133 Milano, Italy.

E-mail: luigi.cocchiarella@polimi.it 
transformation leading, through a symmetry reduction, to perceptual reversal is controlled by the principles of quantum mechanics. A value of the order of $0,32 \mathrm{~mJ} \cdot \mathrm{s}$ is estimated for the elementary neuro-physiological action simulating the Planck constant at a mesoscopic level. In this interdisciplinary study key concepts are utilized: symmetry, symmetry reduction, self-organization, superposition principle and ambiguity.

Igitur impossibile est de aliqua re cognoscere quod fit natura, non cognoscendo motum

Ioannes Duns Scotus Physicorum Aristotelis Quaestiones, Tom. II, Lib. III - 4 (ed. L. Durand, Lyon 1639)

\section{INTRODUCTION}

It is through dynamics that we can learn about the nature of anything. The visual perception of ambiguous images, apparently endowed with some form of oscillatory dynamics, may represent vividly similar physical processes occurring in brain neural networks [Mai05]. Actually an image which induces a dynamical process can be considered as a graphical representation of that process, and may help elucidating the physiology (physics + logic) at the base of perception. The most intelligent living organisms rely on visual perception in order to map and to memorize the objects and movements within their habitat. Information is carried to the vision organs by electromagnetic waves emitted or diffused by the surrounding bodies. Our visual system is based on a combination of (optical) projection and (intelligent) cognition. The cognitive need of extracting the maximum of information from whatever is around us is an existential feature of our nature [CC14]. This need is challenged by ambiguous figures which have two or more possibilities of being interpreted as $3 \mathrm{D}$ objects. In this case the perception can enter a loop, i.e., an oscillatory state with some characteristic frequency. It is argued that these oscillations correspond to a similar dynamic state of the brain neural system involved, and therefore are suitable to shed light on the logic of perception.

Classical paintings of the kinetic art such as those by Franco Grignani and Victor Vasarely stimulate the process of three-dimensionalization via binocular vision. After the pioneering experimental works by the team of $\mathrm{A}$. Borsellino [B72,B77,B82] and the contributions by 
G. Caglioti since the eighties [C83,C86,C94,C95a-c,C02,C14,C15], these processes have been elucidated by numerous experiments on a wide variety of ambiguous two-dimensional (2D) figures. For instance we refer to the illuminating works by Kleinschmidt et al. [Kle98], Vetter et al. [Vet00], Hesselmann et al. [He08], Kornmeier and Bach [Kor12], Conte, Khrennikov, et al. [Con09,Khr10], just to mention a few. We quote in particular H. Atmanspacher et al. [Atm08] who dedicated special attention to the dynamic perception of the Necker cube (NC) [N1832]. In what follows it is shown, with a sequence of elementary examples based on the Necker cube, that the perception of ambiguous graphs can be represented with the tools of quantum logic, which leads to the suggestion that neural networks, in their response to external stimuli, follow as well - though at a mesoscopic level - a quantum logic.

\section{Ambiguous Figures}

The Necker cube (Fig. 1a) is the best known example of planar graph with an inversion symmetry centre which can be perceived as a $3 \mathrm{D}$ object in two different equally probable ways, depending on whether the lower or the upper square is viewed in front and the other in the rear. The two perceptive ways are shown in Fig. 1d and are obtained by removing either the pair of segments or the pair (Fig. 1b). The operations transforming the NC into the new cubes providing an axonometric representation of two $3 \mathrm{D}$ objects is actually a symmetry reduction: the new graphs have lost the original inversion symmetry and acquired an orientation. They symbolically represent the two possible ways of perceiving the $\mathrm{NC}$ and will be represented by two orthogonal quantum states $|0\rangle$ and $|1\rangle$ normalized to unity. Note that the NC acquires one of the two possible meanings via the reduction of symmetry. On the contrary the removal of the pairs or yields two graphs of hard interpretation (Fig. 1c), which maintain the inversion symmetry and are not discernible in the original NC. The symmetry reduction brings in information.

In general terms we can say that perceptual ambiguity is equivalent in the microscopic world to the superposition of quantum states. A possible analytical description of an ambiguous image as a superposition of two graphs is illustrated in Fig. 2: the gradual fading (described 
by sine/cosine factors) of either the $(\beta, \gamma)$ or the $(\alpha, \delta)$ pair leads to the polarized quantum states $|0\rangle$ and $|1\rangle$.

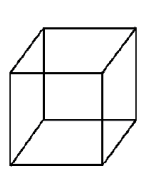

a)

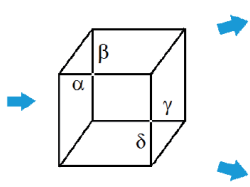

b)
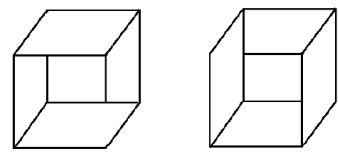

c)

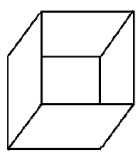

d)

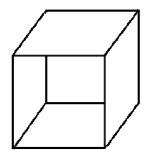

Fig. 1 - (a) The Necker cube (NC) is a planar (2D) graph with inversion symmetry with respect to its center. It can however be perceived as a 3D cube with two equivalent orientations, between which a spontaneous perception oscillation occurs. The equivalent orientations are made after removing the segment pairs $(\beta, \gamma)$ or $(\alpha, \delta)$ shown in $(b)$ : the resulting graphs, shown in (d), have clearly lost the inversion symmetry and are very stable. The NC is viewed as a superposition of two quantum states represented by the two graphs of panel (d). (c) The inversion symmetry is preserved if the segment pairs $(\alpha, \gamma)$ or $(\beta, \delta)$ are removed in $(b)$, but in this case the resulting graphs are visually quite unstable and cannot be discerned in the original NC.

$\cos \frac{\pi}{4}|0\rangle+\sin \frac{\pi}{4}|1\rangle$
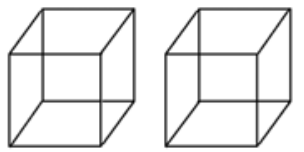

$\sin \frac{\pi}{4}|0\rangle+\cos \frac{\pi}{4}|1\rangle$

$\cos \theta|0\rangle+\sin \theta|1\rangle$
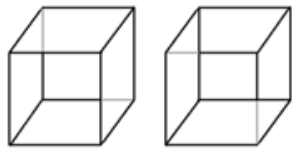

$\sin \theta|0\rangle+\cos \theta|1\rangle$

$\cos (0)|0\rangle+\sin (0)|1\rangle$

$\equiv|0\rangle$
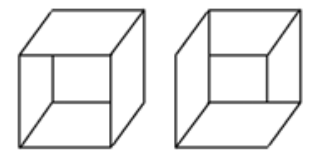

$\cos (0)|0\rangle+\sin (0)|1\rangle$

$\equiv|1\rangle$

Fig. 2 - Illustration of the superposition of two quantum states $|0\rangle$ and $|1\rangle$ (bottom),

leading to the perceptually ambiguous Necker cube (top) via the variation of the mixing angle (co-latitude) $\theta$. 
Another classical example of bistable image is that of vase/face profiles, largely diffused in $18^{\text {th }}-19^{\text {th }}$ century prints, and re-edited by the Danish psychologist Edgar Rubin in his thesis (1915) [Rub15], shown in Fig. 3 in the original version and its black/white complement. The image clearly represents the superposition of two largely inequivalent quantum states.
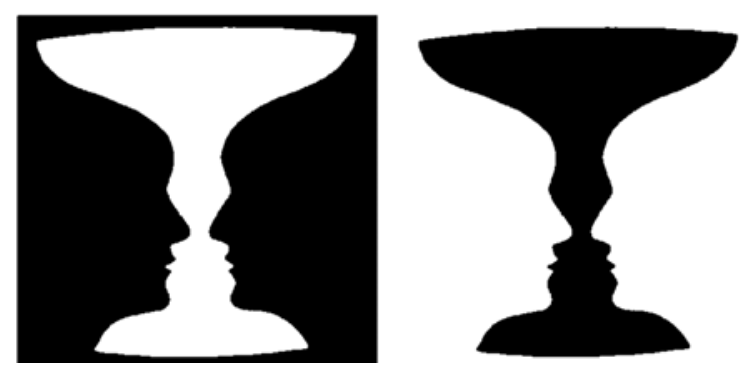

Fig. 3 -Edgar Rubin's vaselfaces ambiguous image shown in two complementary versions which exemplify the prevalent perception of face profiles or that of the vase [Rub15].

There is of course an infinity of ambiguous figures where a plane symmetric figure is perceived as a tridimensional object in two or more different ways. An example, perhaps the simplest one, is a plane representation of a dihedron which can be perceived as either a convex or a concave 3D figure. A sequence of such dihedra, forming, e.g., the Schroeder staircase [Sc858], represented in Fig. 4 in the artistic rendering by Marcello Morandini [Mor15]. The possibility of viewing it as a staircase or as an undulated roof is quite transparent, although the oscillation frequency may be considerably slower than that of a simple Necker cube.

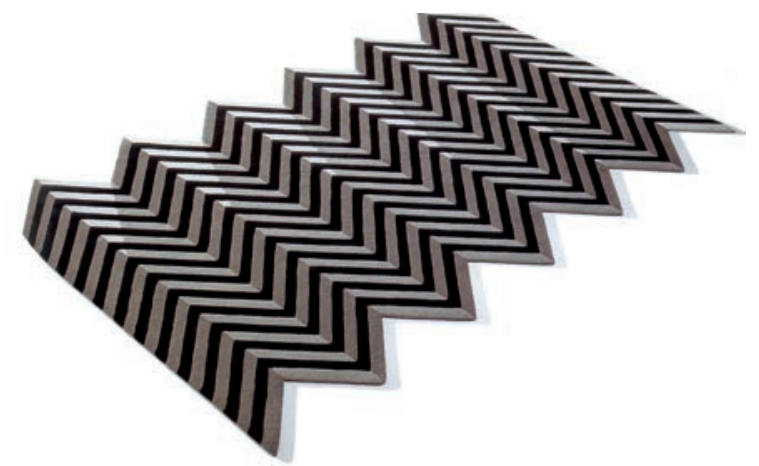

Fig. 4 - The Schroeder staircase [Sc858] in an artistic rendering by Marcello Morandini [Mor15]. 


\section{A QUANTUM REPRESENTATION}

The two states, representing the alternative ways of viewing the NC of Fig. 1 (or each of the two dihedra of Fig. 2), and the oscillations produced by the act of observation find several analogies in the quantum mechanics of two-level systems. The simplest case is that of a particle in a symmetric double well (Fig. 5a), with a tunnel-induced splitting (Fig. 5b) between the eigenvalue $E_{+}$of the symmetric ground state $(|0\rangle+|1\rangle) / \sqrt{2}$ and the eigenvalue $E_{-}$of the upper anti-symmetric state $(|0\rangle-|1\rangle) / \sqrt{ } 2$. When the observation action puts the system in an initial state, say $|0\rangle$, an oscillation of frequency proportional to $E_{-}-E_{+}$between $|0\rangle$ and $|1\rangle$ sets up. The amount of neural energy involved in the perception provides a meaning to the energy splitting, and the proportionality constant $C$ (hereafter called elementary neuro-physiological action) between that physiological energy $E_{r}$ and the observed oscillation frequency is considered as an intrinsic property of the neural system. We consider the Hamiltonian

$$
\hat{\mathrm{H}}=\left|\begin{array}{ll}
E_{0} & E_{r} \\
E_{r} & E_{0}
\end{array}\right|
$$

with $E_{0}=\langle 1|\hat{\mathrm{H}}| 1\rangle=\langle 0|\hat{\mathrm{H}}| 0\rangle \quad$ the eigenvalue for a single well. The timedependent Schrödinger-like equation for the double well

$$
i C \frac{\partial}{\partial t}| \pm\rangle=\hat{\mathrm{H}}| \pm\rangle
$$

has eigenvalues $E=E_{0} \pm E_{r}$ and eigenfunctions

$$
| \pm\rangle=\frac{1}{\sqrt{2}}\left(|0\rangle_{ \pm}|1\rangle\right) \exp \left[-i\left(E_{0} \pm E_{r}\right) t / C\right]
$$

The perception act consists in reducing the symmetry by putting the perceived image in an initial state $\left|\psi_{ \pm}(0)\right\rangle$ evolving like:

$$
\begin{aligned}
& \left|\psi_{+}(t)\right\rangle=\frac{1}{\sqrt{2}}(|+\rangle+|-\rangle)=\exp \left(-i E_{0} t / C\right)\left[\cos \frac{E_{r} t}{C}|0\rangle+i \sin \frac{E_{r} t}{C}|1\rangle\right] \\
& \left|\psi_{-}(t)\right\rangle=\frac{1}{\sqrt{2}}(|+\rangle-|-\rangle)=\exp \left(-i E_{0} t / C\right)\left[\cos \frac{E_{r} t}{C}|0\rangle-i \sin \frac{E_{r} t}{C}|1\rangle\right]
\end{aligned}
$$




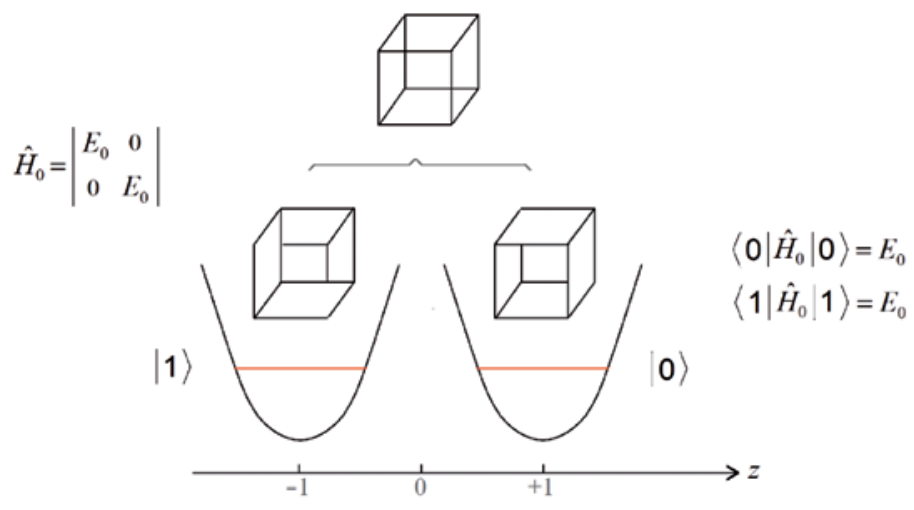

a)

$$
\hat{H}_{0}=\left|\begin{array}{cc}
E_{0} & 0 \\
0 & E_{0}
\end{array}\right|
$$

b)

$$
\hat{H}=\left|\begin{array}{ll}
E_{0} & E_{r} \\
E_{r} & E_{0}
\end{array}\right| \quad \quad E_{ \pm}=E_{0} \pm E_{r} \quad|-\rangle=\frac{1}{\sqrt{2}}(|0\rangle-|1\rangle) \exp \left[-i\left(E_{0}+E_{r}\right) t / C\right]
$$

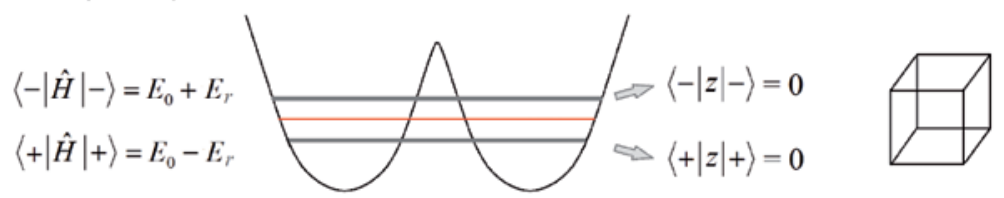

$$
\langle 1|z| 1\rangle=-1 \quad\langle 0|z| 0\rangle=1
$$

$$
|+\rangle=\frac{1}{\sqrt{2}}(|0\rangle+|1\rangle) \exp \left[-i\left(E_{0}-E_{r}\right) t / C\right]
$$

Fig. 5 - (a) A double-well quantum representation of the two degenerate states $|0\rangle$ and $|1\rangle$ in which the NC can be perceived. (b) When the two wells are communicating through a finite barrier, the degeneration is lifted by $2 E_{r}$ yielding two eigenstates $\left.1+\right\rangle$ and

1-) which are symmetric and anti-symmetric superpositions of the original states. When the observation of the NC puts it in the state $|0\rangle$ or $|1\rangle$, an oscillation of frequency $2 E_{r} / C$ starts between these two states.

The perceived configuration at time $t$ is then given by the expectation value $\left\langle\psi_{ \pm}(t)|z| \psi_{ \pm}(t)\right\rangle$, where the coordinate $z$ has the expectation values

$$
\begin{aligned}
& \langle 0|z| 0\rangle=1 \text { corresponding to } \\
& \langle 1|z| 1\rangle=-1 \text { corresponding to }
\end{aligned}
$$


$\langle+|z|+\rangle=\langle-|z|-\rangle=0 \quad$ corresponding to

With these definitions it is found:

$$
\left\langle\psi_{ \pm}(t)|z| \psi_{ \pm}(t)\right\rangle= \pm \cos \frac{2 E_{r} t}{C}
$$

Thus the oscillation between the two equivalent non-stationary states occurs with a frequency $\omega=2 E_{r} / C\left(\operatorname{period} T=\pi C / E_{r}\right)$. The expectation value of the energy for this time-dependent non-stationary state is of course constant and given by

$$
\begin{aligned}
\left\langle\psi_{+}(t)|\hat{\mathrm{H}}| \psi_{+}(t)\right\rangle= & \langle 0|\hat{\mathrm{H}}| 0\rangle \cos ^{2} \frac{E_{r} t}{C}+\langle 1|\hat{\mathrm{H}}| 1\rangle \sin ^{2} \frac{E_{r} t}{C} \\
& -\frac{i}{2}[\langle 0|\hat{\mathrm{H}}| 1\rangle-\langle 1|\hat{\mathrm{H}}| 0\rangle] \sin \frac{2 E_{r} t}{C}=E_{0}
\end{aligned}
$$

and the same for $\psi_{-}(t)$.

Another quantum system providing a useful analogy with the NC representation if a Josephson junction, where the oscillation in the ring is set up by the application of a constant electric bias $V$ across the junction. In the case of a Josephson junction the oscillation frequency is equal to $2 \mathrm{eV} / \mathrm{h}$, whereas for the perception case the proportionality constant is again $C$, provided the applied bias energy is interpreted as the physiological energy, i.e., $E_{r}=e V$.

\section{The ELEMENTARY NEURO-PHySIOLOGICAL ACTION $C$}

The elementary neuro-physiologic action $C$ depends of the perception mechanism and, to some extent, on the observer (specifically of the visual balance between the right eye and left eye). It can be defined as the minimum value of the product of the switching time of the perceptual reversal, $t_{r}$, by the physiological energy $E_{r}$. This energy is twice the energy spent for the initial transition from the initial perception of the 2D symmetric graph, Fig. 1a (ground eigenstate), to the perception of one of the two polarized states, Fig. 1d, from which the 
oscillation starts. Jürgen Kornmeier and Michael Bach [Kor12] in their comprehensive revue assign to the "disambiguation of ambiguous visual information" a typical duration $t_{r}$ of about $50 \mathrm{~ms}$, although "the decision about the perceptual outcome has taken place at least $340 \mathrm{~ms}$ before the observer is able to indicate the consciously perceived reversal manually".

More demanding is the estimation of $E_{r}$. We believe that, to this end, the current research on the spatio-temporal mapping of brain activity [Dal01], integrated by multiple imaging modalities, such as the functional magnetic resonance imaging (fMRI), the electro-enchephalography (EEG), or the magneto-enchephalography (MEG) [Hal05], could provide acceptable answers. An estimation of $E_{r}$ can be obtained from measurements of the visual cortex metabolism $(\sim 7 \mathrm{mg}$ glucose/min for $100 \mathrm{~g}$ of tissue [Phe81]) referred to an estimated functionally activated mass of the visual area V5/MT $(\sim 1 \mathrm{~g})$ [Wal03]: this gives an absorbed power of $0.02 \mathrm{~W}$. When multiplied by the disambiguation time $t_{r}=50 \mathrm{~ms}$ it gives $E_{r}=1 \mathrm{~mJ}$. Considering now a typical oscillation period $T$ between oriented graphs of the order of $2 \mathrm{~s}$, the elementary neuro-physiological action parameter turns out to have an order of magnitude of

$C=E_{r} T / 2 \pi \approx 0.32 \mathrm{~mJ} \mathrm{~s}$.

\subsection{Necker cubes and qubits}

The analogy of ambiguous figures with quantum two-level systems suggests that the pair of degenerate states $(|0\rangle,|1\rangle)$ representing the alternative ways of viewing the $\mathrm{NC}$ of Fig. 1 (or each of the two dihedra of Fig. 2) can be chosen to define the elementary quantum of information, i.e., a qu-bit. A question consequential to this analogy is whether the logic governing visual perception is a quantum logic [BCS04,Nie11]. A possible approach is to study the propagation of a NC qubit (current) along an indefinite NC chain (Fig. 6), thus introducing another time scale, the one describing the run of the observation focus from left to right along the $\mathrm{NC}$ chain.

Once the orientation of the starting $\mathrm{NC}$ on the chain extreme left is chosen, then the NC chain can be rapidly covered with no change in orientation due to the close entanglement of the adjacent NCs. When the chain is closed at the extremities by NCs of opposite 
orientations (reflector, Fig. 6) and the observation focus runs forth and back along the chain, the reflection at each extremity causes a flip of the orientation.

chain

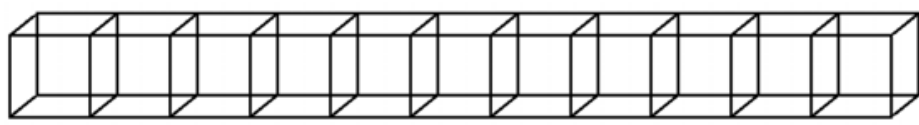

reflector

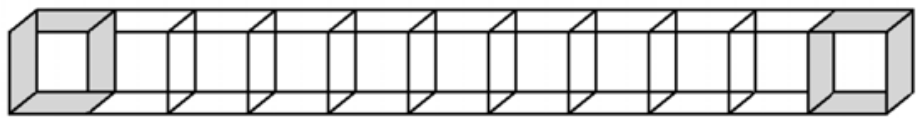

Fig. 6 - Attention runs forth and back along a NC chain introduce another time scale besides that of the oscillation between two opposite perceipts. A NC chain closed by two oriented NCs produces reflections at the extremities with an orientation flip (reflector).

The insertion into a NC chain of an oriented cube, of either $|0\rangle$ and $|1\rangle$ polarization (Fig. 7), transforms an unpolarized current carrying a qubit into a current of either $|0\rangle$ or $|1\rangle$ polarization. The original undefined quantum state represented by one qubit precipitates in one of the two oriented states.

$|0\rangle-$

polarizer

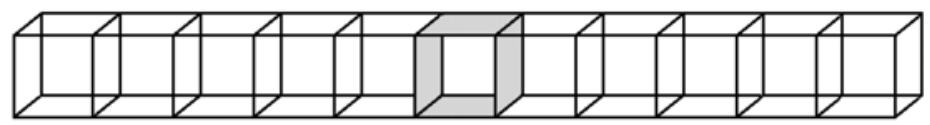

$|0\rangle$

|1>-

polarizer

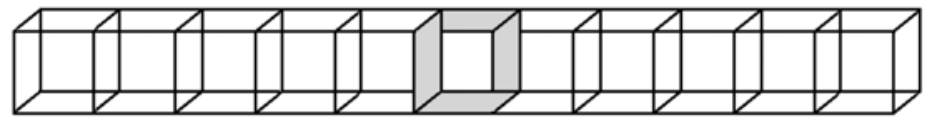

|1>

Fig. 7 - As attention, running along a chain, passes through an oriented NC (polarizer) the polarization, originally undetermined, precipitates in one of the states $|0\rangle$ and $|1\rangle$.

The opposite transformation of a polarized current, either $|0\rangle$ or $|1\rangle$, into a current (qubit) of undefined polarization can occur at the interface (shadowed walls in Fig. 8) between a polarized chain (left) and a NC chain (right). In this way a qubit is generated in the chain, which mimics the function of a Hadamard gate.

A local misfit in the NC period (Fig. 8, phase shift) introduced by a compressed (or elongated) NC, can cause a polarization flip depend- 
ing on the speed of the running attention (current speed defined as the number of NCs scanned per unit time). Ideally it may be argued that a speed of about $1 / 2,1+1 / 2, \ldots$ times the oscillation frequency $\omega$ induces a polarization flip.

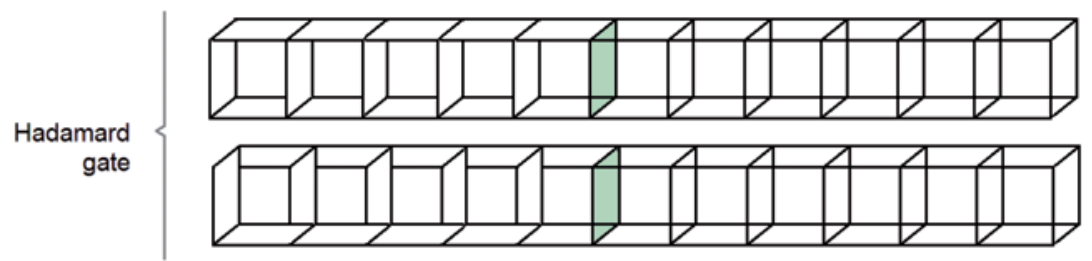

phase shift

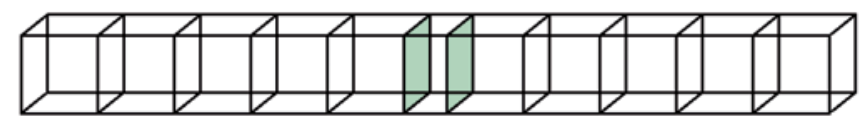

Fig. 8 - The Hadamard gate (shadowed wall), located where a polarized chain turns into a NC chain of undefined polarization, generates a qubit. A local misfit in the NC periodicity (phase shift) can induce a polarization flip depending on the ratio of the two time scales.

Referring to Fig. 9, consider now a given wall in a NC chain which is made either transparent, as any other wall of the chain (labeled by 0 ), or opaque so as to hide segments of the internal horizontal rails of the chain (shadowed area in Fig. 9, labeled by 1). In the first case the current, whatever is its polarization, goes through the wall 0 unchanged, whereas in the second case the wall 1 produces a flip of the polarization: depending on the state of the wall, $|0\rangle$ or $|1\rangle$, the qubit crossing the wall is either unchanged or reversed. This corresponds to the function of a C-not gate.

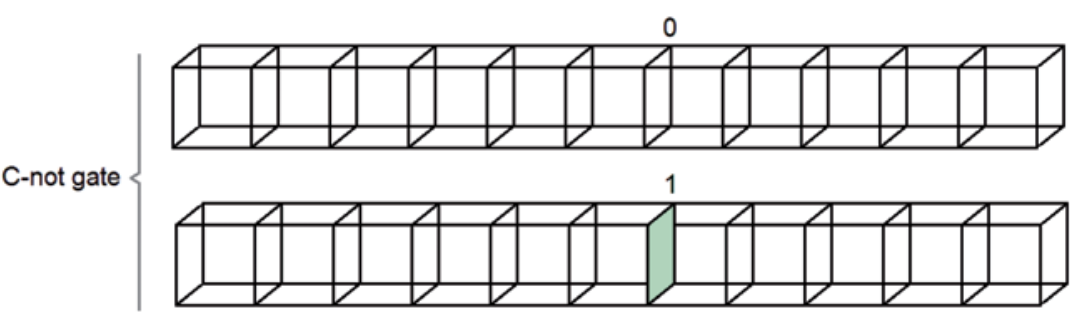

Fig. 9 - A given wall, made either transparent (0) or opaque (1) to the horizontal rails, can work as a C-not gate, leaving unchanged or flipping the predetermined polarization of the running attention. 


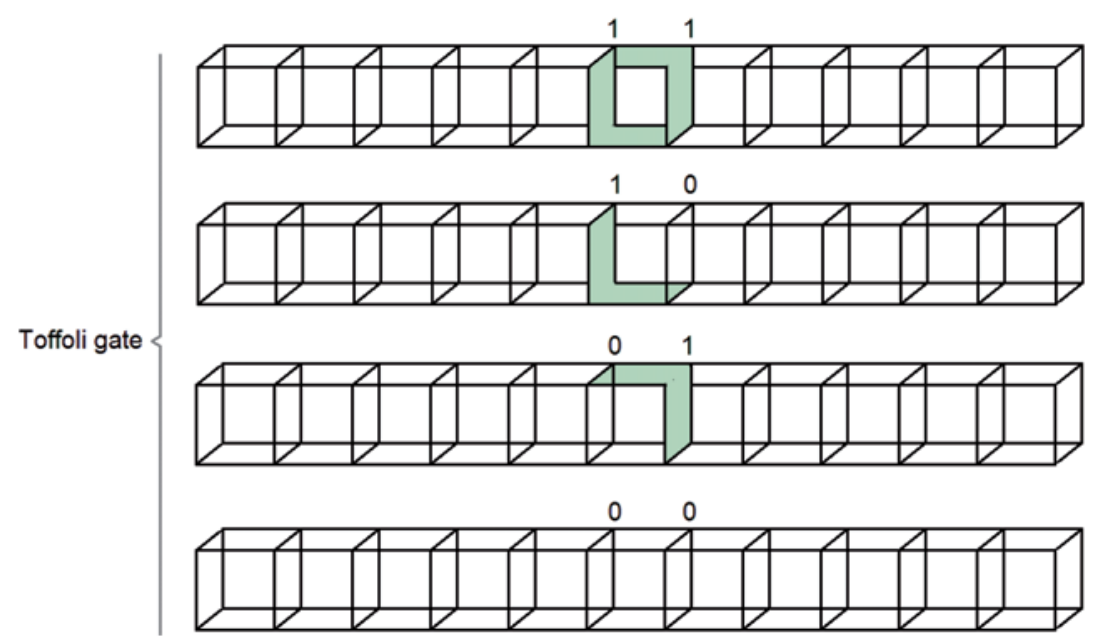

Fig. 10 - L- or reverse L-shaped opaque elements (shadowed areas, labeled 1) are combined with transparent $L$ - or reverse $L$-shaped elements (labeled 0 ) to form a two qubit gate for three qubit operations. The three combinations $|0,1\rangle,|1,0\rangle,|0,0\rangle$ leave the qbit crossing the gate unchanged, while the combination $\mid 1,1>$ produces a flip of the crossing qubit. This corresponds to the function of a Toffoli gate.

A possible realization of a three qubit Toffoli gate is illustrated in Fig. 10. The wanted effect on any arbitrary polarization (qubit) running across the gate is realized with an $\mathrm{L}$ - and a reverse $\mathrm{L}$-shaped element forming the walls of a cube. An opaque (shadowed) element represents a state $|1\rangle$, while a transparent element represents the state $\mid 0>$. In this way the state of the gate is defined by two qubits. Fig. 10 illustrates the four possible states of the gate $|1,1\rangle,|1,0\rangle,|0,1\rangle,|0,0\rangle$ : while the first state produces a flip of the qubit crossing the gate, the other three leave it unchanged.

The above simple realizations of logic functions which alter the perceptual state running along the NC chain are to be considered as a class of possible experiments. There is a virtually infinite number of possible, more complex graphics which could eventually, and more precisely mimic the logic functions. The examples produced above should be considered no more than suggestions, which could however stimulate a new research area having as a goal the demonstration that the visual perception and therefore the related neural network are governed by a quantum logic. It should be noted that the response in the above dynamic experiments, Figs. 6-10, may be largely subjective, 
depending on previous experience or simply on a preliminary information/intuition of what the effect of each gate should be. In the latter case the experiments are a form of learning, i.e., an ad-hoc generation of a quantum-logical software.

\section{CONCLUSIONS}

Under our eyes the Necker cube, internalized in the mind, behaves like a two level quantum system, e.g., as a particle in a double well or a superconducting Josephson ring under a dc bias. Its dynamic perception - an outcome of the mental activity of our many-body neuro-physiological system - culminates in a dynamic instability leading, through a symmetry reduction, to a transformation of the internalized image controlled by the principles of quantum mechanics. On the basis of experimental data obtained from functional NMRI, EEG and MEG we attributed an order of magnitude of $320 \mu \mathrm{J}$ s to the elementary neurophysiologic action $C$. This is a mesoscopic action which has the same meaning and physical dimensions as the Planck constant: it depends on the individual and promotes the symmetry reduction responsible for the perceptual unitary transformation of the twodimensional Necker graph into an oscillating state between two equivalent $3 \mathrm{D}$ percepts.

Nowadays the Schrödinger's sentence [Sch52]: “... we never experiment with just one electron or atom or (small) molecule. In thought experiments, we sometimes assume that we do; this invariably entails ridiculous consequences..." doesn't hold anymore. As remarked by David J. Wineland in his Nobel lecture, these days "we can in fact experiment with individual or small numbers of quantum systems, deterministically preparing superpositions and entangled superpositions" [Win12]. In the present context we tried to demonstrate that the mathematical formalism of quantum mechanics allows to elucidate the neurophysiological process of the dynamic perception of ambiguous images. Moreover suitably organized sets of ambiguous (bistable) graphs may be used for a perceptual simulation of some basic logic functions of the quantum computation. In this way it is hoped that some light may be shed on the logic governing visual perception at the neuro-physiological level. The consequences of applying elementary quantum-mechanical concepts to complex systems like those involved in visual percep- 
tion, where reduction to simplicity relies on coherence, may not be so ridiculous as it was feared by a father of quantum mechanics.

We are aware that our proposed model of quantum-like perception - based essentially on ambiguity and symmetry reduction - should be taken cum grano salis. Regardless of its limits of validity, it is undeniable that our personal mood and our behaviour in society are influenced by our perception. Therefore, with some mixed feeling, we dare to wonder whether, to what extent and in what circumstances our model of a quantum-like perception could be extended, mutatis mutandis, to the way we act and react. Leaving creatures and their communities are much more complex than Helium atoms, photons, Cooper pairs and their boson condensates. However, situations evoking boson statistics and boson condensation can occur in critical situations, e.g. when the survival instinct, a tightly shared objective of vital importance and the tendency of imitation (most likely promoted by the mirror neurons) control, re-orient and unite individuals, damping temporarily their individualism and triggering their solidarity. Think for instance to a shoal of fishes that, at the sudden irruption of a predator, improvise a correlated streaming, think of the shouting of slogans in the political demonstrations or to the emergence of a synchronized, rhythmic applause by an audience that wants to get an encore at the end of an exciting concert. To deepen these topics at this time would expose us to the risk of going beyond the purpose of this work.

\section{REFERENCES}

[Mai05] K. Mainzer, Symmetry and Complexity, The Spirit and Beauty of Nonlinear Science, World Scientific Publishing Co., Singapore, 2005.

[Nie11] M.A. Nielsen and I.L. Chuang, Quantum Computation and Information (Cambridge Univ. Press, 2011).

[BCS04] G. Benenti, G. Casati and G. Strini, Principles of Quantum Computation and Information, Vol 1: Basic Concepts, World Scientific (2004).

[B72] A. Borsellino, A. de Marco, A. Allazetta, S. Rinesi, and R. Bartolini, "Reversal time distribution in the perception of visual ambiguous stimuli", Kybernetik, vol. 10, pp. 139-144, 1972.

[B77] A. Borsellino, F. Carlini, A. De Marco, P. Penengo, A. Trabucco, M. Riani, M.T. Tuccio, Stochastics Model and Fluctations in Reversal Time of Ambiguous Figures", in "Perception" n. 6 (1977), p. 645.

[B82] A. Borsellino, F. Carlini, M. Riani, M.T. Tuccio, A. De Marco, P. Penengo, A. Trabucco "Effects of Visual Angle on Perspectives Reversal for Ambiguous Patterns", in "Perception" n. 11 (1982), p. 263. 
[C83] G. Caglioti. The Dynamics of Ambiguity. Springer, Berlin, Heidelberg, New York, 1992 [available also online]; Symmetriebrechung und Wabrnebmung, with preface by H. Haken. Vieweg, Braunschweig, 1990; Russian Edition, with preface by I. Prigogine. MIR, Moscow, 1997; Japanese Edition, with preface by K. Husimi. Kodansha, Tokyo, 1997; Italian original: Simmetrie infrante nella scienza e nell'arte, with preface by P. Fenoglio, CLUP, Milano, 1983; 2nd Edition, with preface by H. Haken. CittàStudi, Milano, 1994.

[C95a] G. Caglioti. Eidos e Psiche. Struttura della materia e dinamica dell'immagine. Ilisso, Nuoro, 1995; Japanese Edition, Yakuyosha Co. 2001.

[C86] G. Caglioti. The World of Escher and Physics. In M.C. Escher: Art and Science, (H.S.M. Coxeter, M. Emmer, R. Penrose and M.L. Teuber eds), North Holland, Amsterdam, 2871986.

[C95b] G. Caglioti. Perception of Ambiguous Figures: A Qualitative Model Based on Synergetics and Quantum Mechanics, (P. Kruse and M. Stadler, eds). In Ambiguity in Mind and Nature: Multistable Cognitive Phenomena, p. 463. Springer Verlag, Berlin, Heidelberg, New York, 1995.

[C95c] G. Caglioti. From perception to thought, A nonequilibrium dynamic instability implying symmetry reduction. In Katachi U Symmetry, Springer-Verlag, Tokyo, 1995.

[C02] G. Caglioti. Optical art: "illusions" and paradoxes of symmetry and quantum mechanics. In Symmetry 2000 - part 2, I. Hargittai and T.C. Laurent, Eds, pp. 457-466. Portland Press, London, 2002.

[C94] G. Caglioti. The tertium non datur, in Aristotle's Logic and in Physics. J. Mech. Behaviour of Materials, 5, 3: 217-223, 1994. Hills, MI 48331-3535, 2004.

[C15] G. Caglioti. Cognitive Power of Visual Images. In L. Cocchiarella (editor), The Visual Language of Technique: Heritage and Expectations in Research. Vol. 2, Springer (2015, to appear).

[C14] G. Caglioti, L. Cocchiarella, A Eulogy of Ambiguity between Visual Perception and Quantum Mechanics, 16th International Conference on Geometry and Graphics, 4-8 August, 2014, Innsbruck, Austria.

[Con09] E. Conte, A.Y. Khrennikov, O. Todarello, A. Federici, L. Mendolicchio, J.P. Zbilut, Mental states follow quantum mechanics during perception and cognition of ambiguous figures. Open Systems and Information Dynamics 16, 1-17 (2009).

[Khr10] A.Y. Khrennikov, Ubiquitous Quantum Structure: From Psychology to Finance, Springer 2010 (also online).

[Atm08] H. Atmanspacher, M. Bach, T. Filk, J. Kornmeier, H. Römer. Cognitive time scales in a Necker-Zeno model for bistable perception. Open Cybernetics and Systemics Journal 2, 234-251 (2008).

[Kle98] A. Kleinschmidt, C. Büchel, S. Zeki, R.S. Frackowiak, Human brain activity during spontaneously reversing perception of ambiguous figures, Proc. R. Soc. Lond. 265, 2427-2433 (1998).

[Vet00] G. Vetter, J.D. Haynes, and S. Pfaff, Evidence for multistability in the visual perception of pigeons, Vision Research 40 (2000) 2177-2186.

[Hes08] G. Hesselmann, Ch. A. Kell, E. Eger, and A. Kleinschmid, Spontaneous local 
variations in ongoing neural activity bias perceptual decisions, PNAS 105, 10984-10989 (2008).

[Kor12] J. Kornmeier and M. Bach, Ambiguous Figures - What Happens in the Brain When Perception Changes But Not the Stimulus, Front. Hum. Neurosci. 6, 51 (2012).

[Sch52] E. Schrödinger, Are there Quantum Jumps? Part II, British Journal for the Philosophy of Science 3 (11), 233, (1952).

[Win12] D.J. Wineland, Superposition, Entanglement, and Raising Schrödinger's Cat, Nobel Boulder Lecture, December 8, 2012 (published by National Institute of Standards and Technology, Boulder, CO, USA; University of Colorado, Boulder, CO, USA).

[Rub15] E. Rubin, Visual perception of figures: studies in psychological analysis (Gyldendal, Copenhagen 1915); see also (in French): http://figuresambigues. free.fr/ArticlesImage/rubin1. html\#ixzz3nW1D9Drl.

[N1832] L.A. Necker, "Observations on some remarkable optical phaenomena seen in Switzerland; and on an optical phaenomenon which occurs on viewing a figure of a crystal or geometrical solid". London and Edinburgh Philosophical Magazine and Journal of Science 1 (5), 329-337 (1832). doi:10.1080/ 14786443208647909.

[Dal01] A.M. Dale and E. Halgren, Spatiotemporal mapping of brain activity by integration of multiple imaging modalities, Current Opinion in Neurobiology, 11, 202-208 (2001).

[Hal05] Y.O. Halchenko, S.J. Hanson, and B.A. Pearlmutter, Multimodal Integration: fMRI, MRI, EEG, MEG, in L. Landini, V. Positano and M.F. Santarelli (eds) Advanced Image Processing in Magnetic Resonance Imaging, p. 223-265, (Dekker Ser. on Signal Processing and Communications, ISBN 0824725425, 2005).

[Phe81] M.E. Phelps, D.E. Kuhl, and J.C. Mazzotta, Science 211, 1445 (1981).

[Wal03] N.B. Walters, G.F. Egan, J.J. Kril, M. Kean, P. Waley, M. Jenkinson, and J. Watson, PNAS 100, 2981 (2003).

[Sc858] H.G.F. Schroeder (1858). Reproduced in: A.M. Colman, Oxford Dictionary of Psycology, $4^{\text {th }}$ edition (Oxford Univ. Press, 2015) p. 674; and discussed in: A. Scott, Stairway to the Mind: The Controversial New Science of Consciousness (Springer Verlag, 1995) p. 95.

[Mor15] M. Morandini, Sculptures (Aug - 25 Sept 2015 Exhibition at Cortesi Gallery, London): the poster shows a more elaborate version incorporated into a complex multi-ambiguous spiral staircase; see also www.morandinimarcello.com/ it/home 